\title{
Supersymmetric flux backgrounds and generalised special holonomy
}

\author{
André Coimbra \\ IHES, Bures-sur-Yvette, France \\ E-mail: comibralihes.fr

\section{Charles Strickland-Constable*} \\ CEA/Saclay, Gif-sur-Yvette, France, and IHES, Bures-sur-Yvette, France \\ E-mail: charles.strickland-constabledcea.fr
}

\begin{abstract}
Generalised geometry provides an elegant geometrical formulation of the supergravity theories underlying string theory and $\mathrm{M}$ theory. In this contribution, we review recent work showing that general supersymmetric flux backgrounds can be described as the analogue of special holonomy manifolds in this new geometry. The Killing superalgebra, which has a neat manifestation in this language, plays a key role in the proof of the result for $\mathscr{N}>2$ supersymmetry and its form can be fixed explicitly using this technology.
\end{abstract}

Corfu Summer Institute 2016 "School and Workshops on Elementary Particle Physics and Gravity" 31 August - 23 September, 2016

Corfu, Greece

\footnotetext{
* Speaker.
} 


\section{Introduction}

The description of supersymmetric vacua is a problem of central importance in both string phenomenology and the AdS/CFT correspondence (for a review see [1]). In the absence of fluxes the internal geometry of a Minkowski background is forced to be a manifold with special holonomy, such as the celebrated Calabi-Yau spaces [2]. These geometries have an integrable structure, which is a key feature enabling the study of their properties such as their Ricci-flatness and their moduli spaces. However, for geometries which include fluxes, this property is broken; the system can be described as a $G$-structure with non-vanishing intrinsic torsion determined by the fluxes $[3,4,5]$. A further complication is that the structure group $G$ is not determined purely by the number of preserved supersymmetries. These issues make the general classification and analysis of such geometries substantially more involved.

Generalised geometry $[6,7]$ provides a new approach to these problems. In [8] it was shown that four-dimensional supersymmetric Minkowski backgrounds of type II theories correspond to generalised Calabi-Yau manifolds. In particular, they are $S U(3) \times S U(3)$ structures on the $O(6,6)$ generalised tangent space $T \oplus T^{*}$ together with a geometrically natural notion of integrability. This construction effectively includes the NS-NS flux into the geometry of the system, though the integrability is again broken if there are also RR-fluxes. While this rewriting does not fully classify such backgrounds, and does not formulate backgrounds with RR flux as integrable structures, it has nonetheless been applied with great success to the study of supersymmetric solutions $[9,10,11]$.

Later, exceptional generalised geometry was developed $[12,13]$ and it was shown that it is possible to formulate the full internal sector of supergravity in generalised geometry language [14, 15, 16]. The exceptional geometry can include all the fields of M-theory and type II theories in the geometric construction, and for the type II embedding it can be viewed as a direct extension of the original construction of Hitchin and Gualiteri. For example, the generalised tangent space can be viewed as an extension of the $O(d, d)$ generalised tangent space (whose twisting encodes the NS-NS flux) by $O(d, d) \times \mathbb{R}^{+}$generalised tensor bundles [17], with the extension controlled by the remaining fluxes.

Given the earlier results relating supersymmetric backgrounds to integrable generalised structures, it is natural to wonder whether there is a notion of integrable generalised $G$-structure in exceptional generalised geometry which captures the supersymmetry conditions including all possible fluxes. The motivation for this is clear; the generalised geometry formalism provides compact expressions for complicated relations which are powerful tools for the study of general flux backgrounds, and typically an integrable structure allows one to describe features such as the moduli space of a geometry much more easily. Finding the moduli space of flux compactifications is an important problem in string phenomenology and AdS/CFT and, while these questions are beyond the scope of the present article, we remark that some significant steps in these directions have already been made in $[18,19,20]$ using precisely the type of generalised geometry structures we will describe here. It seems very likely that many more such applications will be developed in the future.

The relevant notion of integrability for generalised $G$-structures was introduced in [21]. There, the generalised intrinsic torsion was defined, and its vanishing provides the relevant definition of 
integrability. This was also called generalised special holonomy ${ }^{1}$ by analogy with the Riemannian geometry case where vanishing intrinsic torsion corresponds to special holonomy, and we adopt this language here. If one focuses on the structure defined by a single nowhere vanishing spinor, it was shown in [21] that the integrability of the structure is equivalent to the Killing spinor equations and thus preserved supersymmetry. It was remarked that, in four-dimensional counting, the same derivation also worked for $\mathscr{N}=2$ supersymmetry (shown in detail in [18]), but not for $\mathscr{N} \geq 3$.

In this contribution we will describe recent work [24] showing that the same is true for any number of preserved supersymmetries, as well as reviewing the generalised geometry formalism and the generalised holonomy construction of [21]. We will focus on eleven-dimensional supergravity backgrounds for convenience of presentation, though exactly the same statements hold in the corresponding formulations of type II theories. Our major conclusion is that supersymmetric Minkowski backgrounds are exactly the internal spaces with generalised special holonomy in the relevant stabiliser group. A notable improvement on previous studies is that this provides a single treatment of backgrounds preserving any number $\mathscr{N}$ of supersymmetries.

In order to achieve such a uniform description, we will work largely with spinor quantities, rather than defining the generalised structures in terms of spinor bilinear objects, as was the approach taken in other works in this area $[8,13,25,26,18,19,27]$. However, in the process, we are drawn to consider also the generalised vectors which arise as bilinears of the Killing spinors. These are seen to generate generalised isometries, and together with the Killing spinors they provide a kind of super-isometry algebra on the internal space. This algebra fixes the form of the Killing superalgebra $[28,29]$ of the eleven-dimensional background, of which it forms the internal sector. In particular, the generalised holonomy construction provides a purely geometric proof that the Killing superalgebra of warped Minkowski backgrounds is always the $\mathscr{N}$-extended superPoincaré algebra.

Before we begin the exposition, let us remark that the formalism is capable of describing also supersymmetric AdS flux backgrounds. The integrability condition is simply modified so that a constant singlet intrinsic torsion is switched on, given by the (square root of the) cosmological constant, and this is thought of as analogous to Sasaki-Einstein structures or weak holonomy. In dimensions four and higher, this was shown in [30] for minimally supersymmetric AdS backgrounds, and for AdS backgrounds with 8 supercharges in [19]. We hope to report results for AdS backgrounds corresponding to those given below in future work [31].

\section{Supersymmetric Minkowski backgrounds}

The system of equations describing the supersymmetric backgrounds of interest here are derived as follows. The eleven-dimensional metric is taken to have the standard warped-product form

$$
\mathrm{d} s_{10,1}^{2}=\mathrm{e}^{2 \Delta} \eta_{\mu \nu} \mathrm{d} x^{\mu} \mathrm{d} x^{v}+g_{m n} \mathrm{~d} y^{m} \mathrm{~d} y^{n}
$$

\footnotetext{
${ }^{1}$ Note that in $[22,23]$, the holonomy of the eleven-dimensional supercovariant connection was also referred to as generalised holonomy and on imposing an algebraic constraint on the Killing spinor, the construction of [22, 23] gives rise to the same holonomy groups we will derive here. However, the definition we discuss differs from this approach and is exactly equivalent to preserving supersymmetry, while providing an integrable geometric structure.
} 
In order to maintain the Poincaré symmetry of the Minkowski factor, all fields are taken to depend only on the internal coordinates $y^{m}$ and to be scalars with respect to this group. We are thus able to keep the internal components of the three-form field $A_{(3)}$. which give an internal four-form field strength $F_{(4)}$. Here, we will focus on the case of a four-dimensional Minkowski factor, for convenience of presentation. In this case, one can also keep the four-form field strength on the external space and dualise this to a seven-form field strength on the internal space, which can be associated with a six-form potential via $\tilde{F}_{(7)}=\mathrm{d} \tilde{A}_{(6)}-\frac{1}{2} A_{(3)} \wedge F_{(4)}$. The last term originates from the Chern-Simons coupling in eleven-dimensions.

The eleven-dimensional spinors are decomposed correspondingly as

$$
\varepsilon_{11}=\eta^{+} \otimes \hat{\varepsilon}+\eta^{-} \otimes \hat{\varepsilon}^{*}, \quad \gamma^{(4)} \eta_{ \pm}=\mp \mathrm{i} \eta_{ \pm},
$$

where $\eta$ is a constant external Majorana spinor, and $\hat{\varepsilon}$ is a complex spinor field on the internal space. Requiring that the supersymmetry variation of the eleven-dimensional gravitino vanishes for such a supersymmetry parameter is equivalent to the Killing spinor equations

$$
\begin{aligned}
\mathscr{D}_{m} \hat{\varepsilon}_{i} & :=\nabla_{m} \hat{\varepsilon}_{i}+\frac{1}{288}\left(\gamma_{m}{ }^{n_{1} \ldots n_{4}}-8 \delta_{m}{ }^{n_{1}} \gamma^{n_{2} n_{3} n_{4}}\right) F_{n_{1} \ldots n_{4}} \hat{\varepsilon}_{i}-\frac{1}{12} \frac{1}{6 !} \tilde{F}_{m n_{1} \ldots n_{6}} \gamma^{n_{1} \ldots n_{6}} \hat{\varepsilon}=0, \\
\mathscr{D} \hat{\varepsilon}_{i} & :=\gamma^{m} \nabla_{m} \hat{\varepsilon}_{i}+\gamma^{m}\left(\partial_{m} \Delta\right) \hat{\varepsilon}_{i}-\frac{1}{96} \gamma^{m_{1} \ldots m_{4}} F_{m_{1} \ldots m_{4}} \hat{\varepsilon}_{i}-\frac{1}{4} \frac{1}{7 !} \gamma^{m_{1} \ldots m_{7}} \tilde{F}_{m_{1} \ldots m_{7}} \hat{\varepsilon}=0 .
\end{aligned}
$$

We say that a background preserves $\mathscr{N}$ supersymmetries if there exist $\mathscr{N}$ independent complex spinor fields $\hat{\varepsilon}_{i}, i=1, \ldots, \mathscr{N}$, which are non-vanishing at every point of the internal space, and which solve the Killing spinor equations. This is because such a set of spinors minimally give us $\mathscr{N}$ different ways to uplift the Killing spinors of Minkowski space to eleven-dimensional Killing spinors. We should briefly note that several well-known no-go results exclude the possibility of compact smooth solutions with non-zero fluxes [32, 33, 34]. For the remainder, we consider only the local geometry of the system, and leave global issues such as these, and their possible resolution by including other objects in string theory, for future consideration.

An immediate, but important, consequence of these equations is that the rescaled supersymmetry parameter

$$
\varepsilon=\mathrm{e}^{-\Delta / 2} \hat{\varepsilon},
$$

satisfies [35] (very similar manipulations were also performed in [22, 23])

$$
\tilde{\nabla}_{m} \varepsilon=\nabla_{m} \varepsilon-\frac{1}{4} \frac{1}{3 !} F_{m n p q} \gamma^{n p q} \varepsilon-\frac{1}{4} \frac{1}{6 !} \tilde{F}_{m n_{1} \ldots n_{6}} \gamma^{n_{1} \ldots n_{6}} \varepsilon=0,
$$

Here, $\tilde{\nabla}$ is an $S U(8)$ compatible connection on the spin bundle, as $\left(\gamma^{(2)}, \gamma^{(3)}, \gamma^{(6)}\right)$ give a basis for the $\mathfrak{s u}(8)$ algebra inside $\operatorname{Cliff}(7, \mathbb{R})$. As the Killing spinors are parallel with respect to this connection, all $S U(8)$ invariant inner products of these spinors are constant. In particular, we can find a unitary basis for the space of Killing spinor fields with $\varepsilon_{i}^{\dagger} \varepsilon_{j}=\delta^{i}$.

Another important consequence of the Killing spinor equations, is that the complex vector space of Killing spinors forms a representation of the isometry group of the background, which we define as the group of isometries of the internal metric which also preserve the background fields $\Delta, F_{(4)}$ and $\tilde{F}_{(7)}$. Given a Killing vector $v$ which preserves the background, it can easily be seen that the spinorial Lie derivative along $v$ commutes with the operators $\mathscr{D}_{m}$ and $\mathscr{D}$ appearing in the Killing spinor equations (2.3). Thus, $\mathscr{L}_{v} \varepsilon_{i}$ is another Killing spinor, so there must exist a constant matrix $X_{i}^{j}$ with

$$
\mathscr{L}_{v} \varepsilon_{i}=X_{i}^{j} \varepsilon_{j}
$$




\section{Exceptional generalised geometry for eleven dimensional supergravity}

The internal sector of eleven-dimensional supergravity backgrounds of the type we are considering is described by $E_{7(7)} \times \mathbb{R}^{+}$generalised geometry. This is the generalised geometry on the extended tangent space $[12,13]$ of the seven-dimensional internal space $M$

$$
\begin{aligned}
& E \cong T M \oplus \Lambda^{2} T^{*} M \oplus \Lambda^{5} T^{*} M \oplus\left(T^{*} M \otimes \Lambda^{7} T^{*} M\right), \\
& V=v+\omega+\sigma+\tau,
\end{aligned}
$$

A simple way to explain the appearance of such a geometry in supergravity is to consider the bosonic symmetries of the theory. For our internal sector, these are the seven-dimensional diffeomorphisms of $M$, generated by vector fields, and the gauge transformations of the internal potentials

$$
\begin{aligned}
& A^{\prime}=A+\mathrm{d} \Lambda, \\
& \tilde{A}^{\prime}=\tilde{A}+\mathrm{d} \tilde{\Lambda}-\frac{1}{2} \mathrm{~d} \Lambda \wedge A,
\end{aligned}
$$

which are generated by local two-forms $\Lambda$ and five-forms $\tilde{\Lambda}$. The generators of these symmetries are combined into the generalised vectors $V$. For the precise details, it is important to note that the gauge potentials $A, \tilde{A}$ and gauge parameters $\Lambda, \tilde{\Lambda}$ are defined only locally, while globally these will be twisted by additional gauge transformations between patches of the space. Thus, the generators of the symmetries become global sections of the twisted generalised tangent space, as described in full detail in $[13,15]$.

Though strictly the structure group of $E$ is the parabolic subgroup of $E_{7(7)} \times \mathbb{R}^{+}$which includes the positive roots for the $\mathfrak{g l}(d, \mathbb{R})$ subalgebra, we think of $E$ as being an $E_{7(7)} \times \mathbb{R}^{+}$vector bundle. Associated to this is an $E_{7(7)} \times \mathbb{R}^{+}$principle bundle $\mathscr{F}$ of frames related by a local $E_{7(7)} \times \mathbb{R}^{+}$ transformation to the standard coordinate basis on a patch of the space.

The action of these infinitesimal symmetry transformations on generalised tensors (associated to the principle bundle $\mathscr{F}$ ), is given by the Dorfman derivative, an analogue of the Lie derivative in generalised geometry. Acting on a generalised vector, this can be written out in components as

$$
\begin{gathered}
L_{V} V^{\prime}=\mathscr{L}_{v} v^{\prime}+\left(\mathscr{L}_{v} \omega^{\prime}-i_{v^{\prime}} \mathrm{d} \omega\right)+\left(\mathscr{L}_{v} \sigma^{\prime}-i_{v^{\prime}} \mathrm{d} \sigma-\omega^{\prime} \wedge \mathrm{d} \omega\right) \\
+\left(\mathscr{L}_{v} \tau^{\prime}-j \sigma^{\prime} \wedge \mathrm{d} \omega-j \omega^{\prime} \wedge \mathrm{d} \sigma\right) .
\end{gathered}
$$

It can be thought of as the ordinary Lie derivative, generating the infinitesimal diffeomorphism, together with the appropriate action of $\mathrm{d} \omega$ and $\mathrm{d} \sigma$ via the adjoint of $E_{7(7)} \times \mathbb{R}^{+}$. More abstractly, the Dorfman derivative takes the form

$$
L_{V} V^{\prime}=\partial_{V} V^{\prime}-\left(\partial \times_{\mathrm{ad} \mathscr{F}} V\right) \cdot V^{\prime}
$$

Here the first term is the partial derivative along the vector part $v$, while second term is the derivative of $V$ projected onto the adjoint bundle associated to the frame bundle $\mathscr{F}$. The projection notation $x_{(\ldots)}$ will be widely used in this contribution, and can be thought of simply as a covariant fibre-wise projection onto the specified representation (here the adjoint of $E_{7(7)} \times \mathbb{R}^{+}$).

What we have said so far concerns the bosonic symmetries, we now turn to the bosonic fields themselves. These are encoded as a generalised metric on the generalised tangent space, which is a positive definite inner product, whose local stabiliser group is the maximal compact subgroup 
$S U(8) / \mathbb{Z}_{2} \subset E_{7(7)} \times \mathbb{R}^{+}$. A neat way to construct this explicitly is to use the conformal split frame, which is built from a vielbein for the conventional metric and the other supergravity fields as

$$
\begin{aligned}
\hat{E}_{a}= & \mathrm{e}^{\Delta}\left(\hat{e}_{a}+i_{\hat{e}_{a}} A+i_{\hat{e}_{a}} \tilde{A}+\frac{1}{2} A \wedge i_{\hat{e}_{a}} A\right. \\
& \left.\quad+j A \wedge i_{\hat{e}_{a}} \tilde{A}+\frac{1}{6} j A \wedge A \wedge i_{\hat{e}_{a}} A\right), \\
\hat{E}^{a b} & =\mathrm{e}^{\Delta}\left(e^{a b}+A \wedge e^{a b}-j \tilde{A} \wedge e^{a b}+\frac{1}{2} j A \wedge A \wedge e^{a b}\right), \\
\hat{E}^{a_{1} \ldots a_{5}}= & \mathrm{e}^{\Delta}\left(e^{a_{1} \ldots a_{5}}+j A \wedge e^{a_{1} \ldots a_{5}}\right), \\
\hat{E}^{a, a_{1} \ldots a_{7}} & =\mathrm{e}^{\Delta} e^{a, a_{1} \ldots a_{7}} .
\end{aligned}
$$

This defines an untwisting of the twisted generalised tangent space $E$, since the components of a generalised vector with respect to such a frame provide a globally well-defined vector and two-form etc. The generalised metric can then be defined using the conformal split frame via

$$
G^{-1}=\hat{E}_{a} \otimes \hat{E}^{a}+\hat{E}^{a b} \otimes \hat{E}_{a b}+\hat{E}^{a_{1} \ldots a_{5}} \otimes \hat{E}_{a_{1} \ldots a_{5}}+\hat{E}^{a, a_{1} \ldots a_{7}} \otimes \hat{E}_{a, a_{1} \ldots a_{7}}
$$

where we raise and lower the $S O(d)$ frame indices with $\delta_{a b}$. This is clearly positive definite, and thus stabilised by the maximal compact subgroup $S U(8) / \mathbb{Z}_{2}$ as required. In fact, the frames $\hat{E}_{A}$ satisfying (3.6) form an $S U(8) / \mathbb{Z}_{2}$ principal sub-bundle $\mathscr{P}$ of the frame bundle $\mathscr{F}$, as they are related by local $S U(8) / \mathbb{Z}_{2}$ transformations.

An important ingredient of the generalised geometry construction of supergravity, is the notion of generalised connections. These are defined in exactly the same way as connections on ordinary fibre bundles: they are linear differential operators

$$
\begin{aligned}
D: E & \longrightarrow E^{*} \otimes E \\
D \hat{E}_{A} & =\Omega^{B}{ }_{A} \otimes \hat{E}_{B}
\end{aligned}
$$

where $\Omega^{A}{ }_{B}$ is a local section of $E^{*}$ valued in the adjoint of $E_{7(7)} \times \mathbb{R}^{+}$. Clearly, this can be extended to act on arbitrary generalised tensors via the Leibnitz rule. Using the Dorfman derivative, one can then define the torsion of a generalised connection analogously to the usual torsion of a tangent bundle connection

$$
T(V) \cdot=L_{V}^{(D)}-L_{V}
$$

I.e. the torsion contracted with a generalised vector is the difference of the action of the Dorfman derivative with the connection inserted and the usual Dorfman derivative. The torsion transforms in the $56_{-1}+912_{-1}$ representation of $E_{7(7)} \times \mathbb{R}^{+}$, which is the representation of the embedding tensor [36, 37] of the external space maximal supergravity theory. The precise reasons for this relation to the embedding tensor are given in $[15,38]$.

It is then natural to look for an analogue of the Levi-Civita connection, in generalised geometry. One can indeed define generalised metric compatible connections to be those preserving the generalised metric $G$ and impose the generalised torsion-free condition. While this does fix some components of the connection to be equal to the components of the ordinary Levi-Civita connection and the supergravity fields strengths, it turns out not to fix the connection uniquely.

However, this apparent failure turns out to be irrelevant for describing supergravity. In every case where one wishes to write a supergravity equation using a generalised Levi-Civita connection, 
it turns out that the relevant operation naturally projects out the undetermined components, leaving a unique differential operator.

For an example, consider that one can promote ordinary spinor fields to become sections of the vector bundles with fibres transforming in the $\mathbf{8}$ or $\overline{\mathbf{8}}$ representations of $S U(8)$, which are associated to the $S U(8)$ frame bundle $\mathscr{P}^{2}$. In this way, the Killing spinor equations can be formulated as $S U(8)$ covariant projections of the generalised connection acting on the spinor

$$
\begin{aligned}
\left(D \times{ }_{J} \varepsilon\right)^{[\alpha \beta \gamma]} & =D^{[\alpha \beta} \varepsilon^{\gamma]}=0, \\
\left(D \times{ }_{S} \varepsilon\right)_{\alpha} & =-D_{\alpha \beta} \varepsilon^{\beta}=0 .
\end{aligned}
$$

Here, $S$ and $J$ denote the bundles transforming in the $\mathbf{8}$ and $\mathbf{5 6}$ of $S U(8)$ (see [16] for precise details of this construction). The operators here are precisely such that they are unique in the above sense; they do not depend on the choice of generalised Levi-Civita connection used to evaluate them.

In $[15,16]$ it was shown that the generalised geometry summarised here is able to give a completely natural formulation of the internal sector of eleven-dimensional supergravity. We refer the reader to those papers for further details, and merely comment that the bosonic sector becomes the analogue of Einstein gravity, with the dynamics governed by a generalised Ricci curvature tensor, and the fermionic sector is written using natural $S U(8)$ covariant operators such as those in (3.9). One can perform exactly the same steps in other generalised geometries, leading to other (subsectors of) supergravity theories (see e.g. [39]).

For our study of supersymmetric backgrounds below, it will also be very useful to examine generalised Killing vectors (GKVs). These are generalised vector fields with $L_{V} G=0$, and thus they generate generalised isometries, that is, combined diffeomorphisms and gauge transformations which preserve the metric and the other bosonic supergravity fields. The diffeomorphisms of this type were referred to as isometries of the background in section 2, and they are generated by the vector components of GKVs. Considering this viewpoint, it is simple to show that the conditions for this are that the components of the generalised vector satisfy

$$
\mathscr{L}_{v} g=\mathscr{L}_{v} \Delta=0, \quad \mathscr{L}_{v} A=\mathrm{d} \omega, \quad \mathscr{L}_{v} \tilde{A}=\mathrm{d} \sigma+\frac{1}{2} A \wedge \mathrm{d} \omega,
$$

Similarly to the condition $\nabla_{m} v_{n}=\nabla_{[m} v_{n]}$ for an ordinary Killing vector, this can also be written in terms of the generalised Levi-Civita connection as

$$
\left(D \times \times_{\mathrm{ad} \mathscr{F}} V\right)=\left(D \times{ }_{\mathrm{ad}} \mathscr{P} V\right)
$$

Thus, for a GKV the adjoint action appearing in the Dorfman derivative acts in the adjoint of the $S U(8)$ subgroup of $E_{7(7)} \times \mathbb{R}^{+}$.

It will be very useful to examine the component conditions (3.10) rewritten using the globally defined components of $V$ we obtained using the split frame construction above. However, we will use instead the non-conformal split frame, which differs from (3.5) only by the exclusion of the $\mathrm{e}^{\Delta}$ factors. This is more natural in this context, as the diffeomorphism generated by a generalised vector acts as the Lie derivative by the vector part in the non-conformal split frame. Working in

\footnotetext{
${ }^{2}$ On a spin manifold one can always lift the maximal compact subgroup $S U(8) / \mathbb{Z}_{2}$ to its double cover $S U(8)$.
} 
the non-conformal split frame is also sometimes referred to as working in the untwisted picture of generalised geometry, as in [17]. With respect to this frame, the components will now satisfy

$$
\mathscr{L}_{v} g=\mathscr{L}_{v} \Delta=0, \quad \mathrm{~d} \omega=i_{v} F, \quad \mathrm{~d} \sigma=i_{v} \tilde{F}-\omega \wedge F,
$$

and these relations imply that in this frame, the Dorfman derivative by a GKV reduces to the ordinary Lie derivative by the vector part:

$$
L_{V} V^{\prime}=\mathscr{L}_{v} V^{\prime}
$$

Finally, the Kosmann-Dorfman derivative was introduced in [24]. This provides an analogue of the spinorial Lie derivative (originally introduced by Kosmann [40]) and its action on a spinor $\varepsilon$ is defined using any generalised Levi-Civita connection $D$ as

$$
L_{V}^{\mathrm{KD}} \varepsilon=D_{V} \varepsilon-\left(D \times \times_{\mathrm{ad}} \mathscr{P} V\right) \cdot \varepsilon
$$

Extending by the Leibnitz rule, this can act on any $S U(8)$ tensor. If $V$ is a generalised Killing vector, and we act on a sum of $S U(8)$ objects which carry a natural action of the full $E_{7(7)} \times \mathbb{R}^{+}$, then by (3.11) the Kosmann-Dorfman derivative will agree with the $E_{7(7)} \times \mathbb{R}^{+}$Dorfman derivative. Further, expressed in the non-conformal split frame one has for $V$ a GKV

$$
L_{V}^{\mathrm{KD}} \varepsilon=\mathscr{L}_{v} \varepsilon
$$

exactly as in (3.13), this time with the LHS given by the spinorial Lie derivative. This is a very useful technical result, as it provides an immediate proof of many properties of the KosmannDorfman derivative. For example, for $V, V^{\prime}$ two GKVs, one can immediately see that the algebra closes, i.e.

$$
\left[L_{V}^{\mathrm{KD}}, L_{V^{\prime}}^{\mathrm{KD}}\right] \varepsilon=L_{L_{V} V^{\prime}}^{\mathrm{KD}} \varepsilon,
$$

Following the last paragraph of section 2, one also has that, for $V$ a GKV, the Kosmann-Dorfman derivative preserves the operators (3.9)

$$
L_{V}^{\mathrm{KD}}\left(D \times_{S \oplus J} \zeta\right)=D \times_{S \oplus J}\left(L_{V}^{\mathrm{KD}} \zeta\right) .
$$

\section{An algebra of Killing spinors and Killing spinor bilinears}

We now return to the supersymmetric backgrounds of section 2, and review how the tools we described in section 3 allow us to make some non-trivial general statements concerning generalised isometries induced by the supersymmetry.

Firstly, note that we have already written the Killing spinor equations using generalised LeviCitiva connections in equation (3.9). Consider the complex generalised vectors $V_{i j}$ and $W^{i j}$ built from the Killing spinors via the $S U(8)$ index expressions

$$
\begin{aligned}
\left(V_{i j}\right)^{\alpha \beta} & =\varepsilon_{i}^{[\alpha} \varepsilon_{j}^{\beta]}, & \left(V_{i j}\right)_{\alpha \beta} & =0, \\
\left(W^{i j}\right)^{\alpha \beta} & =0, & \left(W^{i j}\right)_{\alpha \beta} & =\bar{\varepsilon}_{[\alpha}^{i} \bar{\varepsilon}_{\beta]}^{j} .
\end{aligned}
$$


It is straightforward to use (3.9) to check that these satisfy (3.11) and thus they define complex GKVs.

Now consider the Kosmann-Dorfman derivatives of the Killing spinors by these GKVs. An explicit calculation, using (3.9) and $\varepsilon_{i}^{\dagger} \varepsilon_{j}=\delta^{i}{ }_{j}$, reveals that one has

$$
L_{V_{i j}}^{\mathrm{KD}} \varepsilon_{k}=3 D_{V_{[i j}} \varepsilon_{k]}, \quad L_{W^{i j}}^{\mathrm{KD}} \varepsilon_{k}=0 .
$$

Now, from (3.17), one has that there exist constants $X_{i j k}{ }^{l}$ as in (2.6) such that

$$
L_{V_{i j}}^{\mathrm{KD}} \varepsilon_{k}=X_{i j k}^{l} \varepsilon_{l}
$$

and after some further manipulations, one deduces the simple result

$$
L_{V_{i j}}^{\mathrm{KD}} \varepsilon_{k}=0, \quad L_{W^{i j}}^{\mathrm{KD}} \varepsilon_{k}=0 .
$$

This equation says that the generalised isometries generated by the supersymmetries of the background also preserve the Killing spinors. That we were able to prove this completely general result so easily illustrates the power of working in the generalised geometry formalism.

These relations lead to a Lie algebra on the (commuting) internal Killing spinors $\varepsilon_{i}$ and the complex GKVs $V_{i j}$, which takes the form

$$
\left[\varepsilon_{i}, \varepsilon_{j}\right]=V_{i j}, \quad\left[V_{i j}, \varepsilon_{k}\right]=L_{V_{i j}}^{\mathrm{KD}} \varepsilon_{k}=0, \quad\left[V_{i i^{\prime}}, V_{j j^{\prime}}\right]=L_{V_{i i^{\prime}}} V_{j j^{\prime}}=0,
$$

This can be extended by the complex conjugate relations involving $\bar{\varepsilon}^{i}$ and $W^{i j}$, and cross-terms such as $\left[V_{i j}, \bar{\varepsilon}^{k}\right]=L_{V_{i j}}^{\mathrm{KD}} \bar{\varepsilon}^{k}$ which all vanish. We will discuss the implications of this algebra in section 6.

\section{Generalised intrinsic torsion and generalised special holonomy}

Let us now turn to the mathematical characterisation of our supersymmetric backgrounds as generalised structures. Recall that, in the absence of fluxes, the Killing spinor equations reduce to the statement that the Killing spinor is parallel with respect to the Levi-Civita connection, and thus the manifold has special Riemannian holonomy. However, the presence of fluxes breaks this property and one is left instead with a $G$-structure on the tangent bundle of the space with a nonvanishing intrinsic torsion. With this in mind, we begin by reviewing the analogue of such $G$ structures and intrinsic torsion in generalised geometry, following [21, 24].

Firstly, it is clear that the Killing spinors define a $G_{\mathscr{N}}=S U(8-\mathscr{N})$ structure as the $S U(8)$ frames in which the components of $\varepsilon_{i}$ are those of the $i^{\text {th }}$ standard basis vectors in $\mathbb{C}^{8}$ are related by $S U(8-\mathscr{N})$ transformations. These frames define a principle sub-bundle $\mathscr{P}_{\mathcal{N}}$ of $\mathscr{P}$. Note that this is true regardless of the particular form the Killing spinors may take, in contrast to the situation regarding their stabiliser inside $\operatorname{Spin}(7)$, for which there are many possible cases.

Next, the generalised intrinsic torsion is defined, exactly mirroring the ordinary definition. If we let $W$ be the bundle in which the generalised torsion transforms, and let $K_{S U(8)}=E^{*} \otimes$ ad $\mathscr{P}$ be the bundle of differences of generalised connections, then there is a natural mapping

$$
\begin{aligned}
\tau: K_{S U(8)} & \longrightarrow W \\
\delta \Omega & \longmapsto \tau(\delta \Omega)=T\left(D^{\prime}\right)-T(D),
\end{aligned}
$$


where $D$ and $D^{\prime}$ are any two generalised connections with $\delta \Omega=D^{\prime}-D$. Now consider the subbundle $K_{\mathscr{N}}=E^{*} \otimes$ ad $\mathscr{P}_{\mathscr{N}}$ It could be that the image of $\tau$ restricted to $K_{\mathscr{N}}$ is not the whole of $W$ so, labelling $W_{\mathscr{N}}=\left.\operatorname{Im} \tau\right|_{K_{\mathscr{N}}}$, the bundle of the generalised intrinsic torsion is defined to be [21]

$$
W_{\mathrm{int}}=\frac{W}{W_{\mathscr{N}}} .
$$

The torsion of any $G_{\mathscr{N}}$ compatible connection naturally projects onto a section of the bundle $W_{\text {int }}$. This section is the generalised intrinsic torsion, and it is independent of the choice of $G_{\mathscr{N}}$ compatible connection by construction. The generalised intrinsic torsion can be defined similarly for any generalised $G$-structure, though here we are concerned only with the structure groups $G_{\mathscr{N}}$.

This abstract notion can be realised concretely in the following way. Given a $G_{\mathscr{N}}$ compatible connection $\hat{D}$, that is one with $\hat{D} \varepsilon_{i}=0$, we can write this as

$$
\hat{D}=D+\Sigma,
$$

where $D$ is a generalised Levi-Civita connection and $\Sigma$ is a section of $K_{S U(8)}$. As $D$ is torsion-free, the torsion of $\hat{D}$ is equal to $\tau(\Sigma)$. In fact, via a linear algebra calculation, one can show that the intrinsic torsion is captured precisely by the components

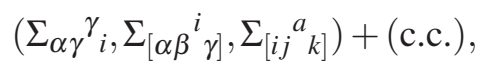

where we have split the $S U(8)$ indices $\alpha, \beta=1, \ldots, 8$ as $\alpha=(i, a)$ where the directions $i, j=$ $1, \ldots, \mathscr{N}$ run along the Killing spinors and $a, b$ label the remaining directions.

Let us examine what the Killing spinor equations imply for the generalised intrinsic torsion. Inserting (5.3) into (3.9), we can express the equations purely as constraints on the components of $\tau(\Sigma)$

$$
\left.D_{[\alpha \beta} \bar{\varepsilon}_{\gamma]}^{i}=-\Sigma_{[\alpha \beta}{ }^{i} \gamma\right]=0, \quad D_{\alpha \beta} \varepsilon_{i}^{\beta}=\Sigma_{\alpha \beta}{ }^{\beta}{ }_{i}=0 .
$$

The Killing spinor equations thus set almost all of the generalised intrinsic torsion to zero. However, naively it appears that they do not constrain the last piece $\left.\Sigma_{[i j}{ }^{a} k\right]$. This piece can be non-zero only for $\mathscr{N} \geq 3$, so for $\mathscr{N} \leq 2$ the Killing spinor equations do immediately imply that the generalised intrinsic torsion vanishes [21, 18].

In Riemannian geometry, a $G$-structure on the tangent bundle (with $G \subset S O(d)$ ) has vanishing intrinsic torsion if and only if the Levi-Civita connection has special holonomy. By analogy with this situation, generalised special holonomy is defined by the vanishing of the intrinsic torsion of a $G$-structure on the generalised tangent bundle [21]. Thus, for $\mathscr{N} \leq 2$ the above already shows that supersymmetric Minkowski backgrounds of eleven-dimensional supergravity have $S U(8-\mathscr{N})$ generalised special holonomy.

But what of the $\mathscr{N} \geq 3$ cases? It would be unsatisfactory to classify such backgrounds using a mathematical notion which only applied to some cases. Let us reexamine equation (4.2), and express those spinor trilinear quantities in terms of the components of $\tau(\Sigma)$. In particular, we find

$$
\left(L_{V_{i j}}^{\mathrm{KD}} \varepsilon_{k}\right)^{a}=\frac{3}{32} \Sigma_{[i j k]}{ }^{a} k,
$$

so that this captures precisely the components of the intrinsic torsion (5.4) which did not appear directly in the Killing spinor equations. However, equation (4.4) already showed that this quantity 
vanishes for our supersymmetric backgrounds. Therefore, we find that the Killing spinor equations do in fact imply that the full intrinsic torsion vanishes in all cases!

Thus, the conclusion is that warped supersymmetric Minkowski backgrounds of eleven-dimensional supergravity are exactly the spaces with generalised special holonomy in the sense defined above. This result can be shown to hold for all external dimensions $d_{\mathrm{ext}} \geq 4$, and the generalised holonomy groups are uniquely fixed by the amount of preserved supersymmetry as listed in table 1. Further, this also applies to the descriptions of type IIA and type IIB vacua in $E_{d(d)} \times \mathbb{R}^{+}$generalised geometry language.

\begin{tabular}{llll}
$d_{\mathrm{ext}}$ & $E_{d(d)}$ & $\tilde{H}_{d}$ & $G_{\mathscr{N}}$ \\
\hline 4 & $E_{7(7)}$ & $\operatorname{SU}(8)$ & $S U(8-\mathscr{N})$ \\
5 & $E_{6(6)}$ & $U S p(8)$ & $U S p(8-2 \mathscr{N})$ \\
6 & $\operatorname{Spin}(5,5)$ & $\operatorname{USp}(4) \times U S p(4)$ & $U S p\left(4-2 \mathscr{N}_{+}\right) \times U S p\left(4-2 \mathscr{N}_{-}\right)$ \\
7 & $\operatorname{SL}(5, \mathbb{R})$ & $U S p(4)$ & $U S p(4-2 \mathscr{N})$
\end{tabular}

Table 1: Generalised structure groups $G_{\mathcal{N}}$ for $d_{\text {ext }}$-dimensional Minkowski backgrounds of type II and eleven-dimensional supergravity preserving $\mathscr{N}$ supersymmetries. When $d_{\text {ext }}=6$ we can have two chiralities of supersymmetry so $\mathscr{N} \rightarrow\left(\mathscr{N}_{+}, \mathscr{N}_{-}\right)$.

\section{Implications for the Killing superalgebra}

The relations in (4.5) are highly reminiscent of those between the supercharges and the momentum in a Poincaré supersymmetry algebra. Below, we will explain how they are indeed closely linked to the Killing superalgebra of the eleven-dimensional background, which we briefly review.

For a generic supersymmetric solution of eleven-dimensional supergravity, one can define a superalgebra on the Killing spinors $\varepsilon$ and Killing vectors $v$ of the background via the following bracket definitions [28, 29]

$$
\begin{aligned}
{\left[\varepsilon_{1}, \varepsilon_{2}\right\} } & =v\left(\varepsilon_{1}, \varepsilon_{2}\right), \\
{[v, \varepsilon\} } & =\mathscr{L}_{v} \varepsilon, \\
{\left[v_{1}, v_{2}\right\} } & =\mathscr{L}_{v_{1}} v_{2},
\end{aligned}
$$

where $\mathscr{L}_{v}$ is the (spinorial) Lie derivative. It is a non-trivial task to check the super-Jacobi-identities for these brackets, and for this derivation we refer to [28, 29].

Here, we merely wish to outline the implications of (4.5) for this algebra in eleven-dimensions. To do this, we simply express the eleven-dimensional objects in terms of our internal objects, and calculate the algebra. Thus, we choose a basis of constant external Weyl spinors $\left\{\eta_{\alpha}\right\}$ and define the (complex) eleven-dimensional spinors

$$
Q_{i, \alpha}=\eta_{\alpha} \otimes \hat{\varepsilon}_{i}, \quad \bar{Q}_{i}^{\dot{\alpha}}=\bar{\eta}^{\dot{\alpha}} \otimes \hat{\varepsilon}_{i}^{c},
$$

The real and imaginary parts of these objects provide a basis for the eleven-dimensional Killing spinors, but we leave them as the complex representation for notational convenience. We then define the vectors $z_{i j}$ to be the vector parts of the generalised vectors $V_{i j}$, in the non-conformal split 
frame, pushed forward into the eleven-dimensional space. The internal algebra (4.5) can be seen to imply that the brackets (6.1) become

$$
\begin{aligned}
{\left[Q_{i, \alpha}, \bar{Q}_{j, \dot{\beta}}\right\} } & =\delta_{i j}\left(\sigma^{\mu}\right)_{\alpha \dot{\beta}} \frac{\partial}{\partial x^{\mu}}, \\
{\left[Q_{i, \alpha}, Q_{j, \beta}\right\} } & =\varepsilon_{\alpha \beta} z_{i j}, \\
{\left[\bar{Q}_{i, \dot{\alpha}}, \bar{Q}_{j, \dot{\beta}}\right\} } & =\varepsilon_{\dot{\alpha} \dot{\beta}} \bar{z}_{i j},
\end{aligned}
$$

with all other brackets vanishing. Thus, we have provided a geometric proof that the Killing superalgebra of a generic four-dimensional Minkowski flux background is the corresponding $\mathscr{N}$ extended super-Poincaré algebra. The central charges become the (generalised) isometries associated to the GKVs $V_{i j}$, and crucially (4.5) shows that these are indeed central in the algebra.

\section{References}

[1] M. Grana, "Flux compactifications in string theory: A Comprehensive review," Phys. Rept. 423, 91 (2006) [hep-th/0509003].

[2] P. Candelas, G. T. Horowitz, A. Strominger and E. Witten, "Vacuum Configurations for Superstrings," Nucl. Phys. B 258, 46 (1985).

[3] J. P. Gauntlett, D. Martelli, S. Pakis and D. Waldram, “G structures and wrapped NS5-branes," Commun. Math. Phys. 247 (2004) 421 [hep-th/0205050].

[4] J. P. Gauntlett and S. Pakis, “The Geometry of $D=11$ Killing Spinors,” JHEP 0304 (2003) 039 [hep-th/0212008].

[5] D. Martelli and J. Sparks, "G structures, fluxes and calibrations in M theory," Phys. Rev. D 68 (2003) 085014 [hep-th/0306225].

[6] N. Hitchin, “Generalized Calabi-Yau manifolds," Quart. J. Math. 54, 281 (2003) doi:10.1093/qjmath/54.3.281 [math/0209099 [math-dg]].

[7] M. Gualtieri, "Generalized complex geometry," Oxford University DPhil thesis (2004) [arXiv:math.DG/0401221] and [arXiv:math.DG/0703298].

[8] M. Grana, R. Minasian, M. Petrini and A. Tomasiello, "Supersymmetric backgrounds from generalized Calabi-Yau manifolds,” JHEP 0408 (2004) 046 [arXiv:hep-th/0406137].

M. Grana, R. Minasian, M. Petrini and A. Tomasiello, "Generalized structures of $N=1$ vacua," JHEP 0511 (2005) 020 [arXiv:hep-th/0505212].

[9] M. Grana, R. Minasian, M. Petrini and A. Tomasiello, "A Scan for new N=1 vacua on twisted tori," JHEP 0705 (2007) 031 [hep-th/0609124].

[10] D. Andriot, "New supersymmetric flux vacua with intermediate SU(2) structure," JHEP 0808 (2008) 096 [arXiv:0804.1769 [hep-th]].

[11] F. Apruzzi, M. Fazzi, D. Rosa and A. Tomasiello, “All $A d S_{7}$ solutions of Type II supergravity,” JHEP 1404 (2014) 064 [arXiv:1309.2949 [hep-th]].

F. Apruzzi, M. Fazzi, A. Passias, D. Rosa and A. Tomasiello, “AdS $S_{6}$ solutions of Type II supergravity," arXiv:1406.0852 [hep-th].

F. Apruzzi, M. Fazzi, A. Passias and A. Tomasiello, "Supersymmetric AdS 5 solutions of massive IIA supergravity,” JHEP 1506, 195 (2015) [arXiv:1502.06620 [hep-th]].

A. Rota and A. Tomasiello, "AdS 4 compactifications of $\mathrm{AdS}_{7}$ solutions in type II supergravity," JHEP 1507, 076 (2015) [arXiv:1502.06622 [hep-th]]. 
[12] C. M. Hull, “Generalised Geometry for M-Theory," JHEP 0707, 079 (2007) [arXiv:hep-th/0701203].

[13] P. P. Pacheco, D. Waldram, "M-theory, exceptional generalised geometry and superpotentials," JHEP 0809, 123 (2008). [arXiv:0804.1362 [hep-th]].

[14] A. Coimbra, C. Strickland-Constable and D. Waldram, "Supergravity as Generalised Geometry I: Type II Theories," JHEP 1111, 091 (2011) [arXiv:1107.1733 [hep-th]].

[15] A. Coimbra, C. Strickland-Constable and D. Waldram, " $E_{d(d)} \times \mathbb{R}^{+}$generalised geometry, connections and M theory," JHEP 1402, 054 (2014) [arXiv:1112.3989 [hep-th]].

[16] A. Coimbra, C. Strickland-Constable and D. Waldram, "Supergravity as Generalised Geometry II: $E_{d(d)} \times \mathbb{R}^{+}$and M theory," JHEP 1403, 019 (2014) [arXiv:1212.1586 [hep-th]].

[17] D. Cassani, O. de Felice, M. Petrini, C. Strickland-Constable and D. Waldram, "Exceptional generalised geometry for massive IIA and consistent reductions," JHEP 1608, 074 (2016) [arXiv:1605.00563 [hep-th]].

[18] A. Ashmore and D. Waldram, "Exceptional Calabi-Yau spaces: the geometry of $\mathscr{N}=2$ backgrounds with flux," arXiv:1510.00022 [hep-th].

[19] A. Ashmore, M. Petrini and D. Waldram, "The exceptional generalised geometry of supersymmetric AdS flux backgrounds," arXiv:1602.02158 [hep-th].

[20] A. Ashmore, M. Gabella, M. Graña, M. Petrini and D. Waldram, "Exactly marginal deformations from exceptional generalised geometry," arXiv:1605.05730 [hep-th].

[21] A. Coimbra, C. Strickland-Constable and D. Waldram, "Supersymmetric Backgrounds and Generalised Special Holonomy,” Class. Quant. Grav. 33, no. 12, 125026 (2016) [arXiv:1411.5721 [hep-th]].

[22] M. J. Duff and J. T. Liu, "Hidden space-time symmetries and generalized holonomy in M theory," Nucl. Phys. B 674, 217 (2003) [hep-th/0303140].

[23] C. Hull, "Holonomy and symmetry in M theory," hep-th/0305039.

[24] A. Coimbra and C. Strickland-Constable, "Supersymmetric Backgrounds, the Killing Superalgebra, and Generalised Special Holonomy,” JHEP 1611, 063 (2016) [arXiv:1606.09304 [hep-th]].

[25] M. Grana, J. Louis, A. Sim, D. Waldram, " $E_{7(7)}$ formulation of $N=2$ backgrounds," JHEP 0907, 104 (2009). [arXiv:0904.2333 [hep-th]].

[26] M. Grana, F. Orsi, “N=1 vacua in Exceptional Generalized Geometry,” JHEP 1108, 109 (2011) [arXiv:1105.4855 [hep-th]].

M. Grana and F. Orsi, “N=2 vacua in Generalized Geometry,” JHEP 1211, 052 (2012) [arXiv:1207.3004 [hep-th]].

[27] M. Graña and P. Ntokos, "Generalized geometric vacua with eight supercharges," JHEP 1608, 107 (2016) [arXiv:1605.06383 [hep-th]].

[28] J. M. Figueroa-O'Farrill, P. Meessen and S. Philip, "Supersymmetry and homogeneity of M-theory backgrounds," Class. Quant. Grav. 22, 207 (2005) [hep-th/0409170].

[29] J. M. Figueroa-O'Farrill, E. Hackett-Jones and G. Moutsopoulos, "The Killing superalgebra of ten-dimensional supergravity backgrounds," Class. Quant. Grav. 24, 3291 (2007) doi:10.1088/0264-9381/24/13/010 [hep-th/0703192].

[30] A. Coimbra and C. Strickland-Constable, "Generalised Structures for $\mathscr{N}=1$ AdS Backgrounds," JHEP 1611, 092 (2016) [arXiv:1504.02465 [hep-th]]. 
[31] A. Coimbra and C. Strickland-Constable, work in progress

[32] P. Candelas and D. J. Raine, "Spontaneous Compactification and Supersymmetry in $d=11$ Supergravity," Nucl. Phys. B 248, 415 (1984).

P. Candelas, "Compactification and Supersymmetry of Chiral $N=2 D=10$ Supergravity," Nucl. Phys. B 256, 385 (1985).

[33] J. M. Maldacena and C. Nunez, "Supergravity description of field theories on curved manifolds and a no go theorem,” Int. J. Mod. Phys. A 16, 822 (2001) [hep-th/0007018].

[34] S. Ivanov and G. Papadopoulos, "A No go theorem for string warped compactifications," Phys. Lett. B 497, 309 (2001) [hep-th/0008232].

[35] M. Gabella, D. Martelli, A. Passias and J. Sparks, “ $\mathscr{N}=2$ supersymmetric $\mathrm{AdS}_{4}$ solutions of M-theory,” Commun. Math. Phys. 325 (2014) 487 [arXiv:1207.3082 [hep-th]].

[36] B. de Wit, H. Samtleben, M. Trigiante, "On Lagrangians and gaugings of maximal supergravities," Nucl. Phys. B655, 93-126 (2003). [hep-th/0212239];

B. de Wit, H. Samtleben, M. Trigiante, “The Maximal $D=4$ supergravities," JHEP 0706, 049 (2007). [arXiv:0705.2101 [hep-th]].

[37] A. Le Diffon, H. Samtleben, M. Trigiante, “ $N=8$ Supergravity with Local Scaling Symmetry," JHEP 1104, 079 (2011). [arXiv:1103.2785 [hep-th]].

[38] K. Lee, C. Strickland-Constable and D. Waldram, "Spheres, generalised parallelisability and consistent truncations," arXiv:1401.3360 [hep-th].

[39] C. Strickland-Constable, "Subsectors, Dynkin Diagrams and New Generalised Geometries," arXiv:1310.4196 [hep-th].

[40] Y. Kosmann, "Dérivées de Lie des spineurs," Ann. di Matematica Pura ed Applicata 91, (1971) 317-395 